\title{
Redes de aprendizaje intraorganizativo: análisis exploratorio de un caso
}

\author{
Sánchez de Pablo González del Campo, Jesús David * \\ Škerlavaj, Miha ** \\ Guadamillas Gómez, Fátima *** \\ Dimovski, Vlado ****
}

\begin{abstract}
Resumen
La importancia del aprendizaje organizacional en la determinación del resultado de la empresa ha provocado un incremento en su análisis por parte de los investigadores. Sin embargo, el modo en el que el aprendizaje se produce en el seno de una empresa constituye una de las principales lagunas existentes en la investigación. Por ese motivo, el objetivo de este trabajo es identificar los aspectos más relevantes que fomentan el aprendizaje organizativo de una empresa. Así, realizamos un estudio exploratorio, por el que por medio de un cuestionario los integrantes de una empresa identificaron a sus compañeros de los que más aprenden. Consideramos que la amplia experiencia de los trabajadores en la empresa es la variable clave para que el resto de empleados tengan incentivos para aprender de ellos y convertirse de este modo en trabajadores clave en la red de aprendizaje intraorganizativo. De este modo, la empresa debe motivar a sus trabajadores con mayor experiencia para que se sientan identificados con la organización y estén dispuestos a compartir su conocimiento con sus compañeros. Así, se incrementará el aprendizaje organizativo y, por lo tanto, su resultado.
\end{abstract}

Palabras clave: Aprendizaje organizacional, redes sociales, red de aprendizaje intraorganizativo.

Recibido: 20-07-08. Aceptado: 10-12-09

* Doctor en Administración y Dirección de Empresas, Profesor Ayudante Doctor, Departamento de Administración de Empresas, Facultad de Ciencias Químicas, Universidad de Castilla-La Mancha, España. E-mail: jesusdavid.sanchez@uclm.es. Autor para la correspondencia.

** Doctor en Gestión y Finanzas, Profesor Ayudante, Departamento de Gestión y Organización, Universidad de Ljubljana, Eslovenia. E-mail: miha.skerlavaj@ef.uni-lj.si.

*** Doctora en Ciencias Económicas y Empresariales, Profesor Titular, Departamento de Administración de Empresas, Facultad de Ciencias Jurídicas y Sociales, Universidad de Castilla-La Mancha, España. E-mail: Fatima.Guadamillas@uclm.es.

**** Doctor en Gestión y Finanzas, Profesor Titular, Departamento de Gestión y Organización, Facultad de Economía, Universidad de Ljubljana, Eslovenia. E-mail: vlado.dimovski@ef.uni-lj.si. 
Redes de aprendizaje intraorganizativo: análisis exploratorio de un caso

Sánchez, Jesús D.; Škerlavaj, Miha; Guadamillas Gómez, Fátima y Dimovski, Vlado

\title{
Intraorganizational Learning Networks: Exploratory Analysis of a Case
}

\begin{abstract}
The importance of organizational learning for business performance has caused an increase in its analysis by researchers. However, the way that learning takes place in a firm constitutes one of the main research gaps. The aim of this study is to identify the most relevant aspects that foster organizational learning in a firm. An exploratory study was made using a questionnaire on which employees identified the coworkers from whom they learn most. The authors consider that broad employee experience in the firm is the key variable for incentivating other employees to learn from them, turning these employees into key workers in the intraorganizational learning network. Therefore, the firm should motivate their more experienced workers so that they identify with the organization and are willing to share their knowledge with their coworkers. Thus, organizational learning and the firm's performance will increase.
\end{abstract}

Key words: Organizational learning, social networks, intraorganizational learning networks, experience, central position.

\section{Introducción}

El aprendizaje que se produce de forma interna en una empresa surge de la interacción entre sus empleados. Cuando estas relaciones se repiten en el tiempo se comienzan a formar estructuras de red (Wasserman y Faust, 1994). El enfoque que analiza las características de dichas redes es el enfoque de redes sociales. En este artículo tratamos de analizar las relaciones de aprendizaje, por lo que dentro del enfoque de redes estudiaremos las redes de aprendizaje intraorganizativo.

El objetivo es explorar como sucede el aprendizaje a nivel intraorganizacional y analizar las características personales entre quienes se dan esas relaciones. Así se obtendrá información sobre los factores que pueden fomentarlo. De este modo, los gerentes podrán potenciar el aprendizaje en sus empresas y hacer frente de forma más efectiva a los problemas económicos globales. Se realizará el estudio de un caso para exponer su modo de creación y organización de conocimiento y analizar las características principales de su red de aprendizaje.

Con ese fin se elaboró un cuestionario donde se pedía a los 240 empleados que identificaran a los compañeros de los que más habían aprendido. Posteriormente, se realizaron entrevistas personales con varios trabajadores de la empresa de diferentes niveles jerárquicos. Se confirmó un alto ajuste entre las opiniones subjetivas de la entrevista y los resultados obtenidos a través de los cuestionarios.

Entre los empleados que no respondieron nos encontramos con que un número importante de los mismos llevaban poco tiempo trabajando en la empresa. Por tanto, no tenían perspectiva suficiente para completar la mayor parte de los ítems del cuestionario. De este modo, tras reducir la muestra nos quedamos con 209 trabajadores con una permanencia en la empresa de al menos tres meses. Al final del proceso de recolección de datos obtuvimos 175 cuestionarios cum- 
plimentados lo que representa una tasa de respuesta del 83,73\%. El software empleado para el análisis de datos fue Pajek 1.04 (Batagelj y Mrvar, 2005; de Nooy, Mrvar y Batagelj, 2005).

Para lograr nuestros objetivos, comenzaremos analizando el enfoque de redes sociales centrándonos posteriormente en las redes de aprendizaje organizativo. Para ello será necesario el estudio de las diferentes perspectivas de aprendizaje organizacional. A continuación, realizaremos el estudio de una red de aprendizaje intraorganizativo para identificar sus aspectos más relevantes.

\section{Aprendizaje organizacional desde el enfoque de redes sociales}

Las redes sociales están formadas por vínculos entre un grupo definido de personas, con la propiedad de que las características de estos vínculos podrían ser empleadas para interpretar el comportamiento social de las personas implicadas (Mitchell, 1969). Desde este enfoque, las empresas se constituyen como un sistema de personas y grupos unidos por una variedad de relaciones, que no se presentan de forma directa entre todos sus miembros.

El análisis de redes sociales trata de estudiar la estructura y las pautas de estas relaciones y busca identificar para ambos casos sus causas y consecuencias (Tichy, Tushman y Fombrun, 1979). No incide en las características individuales de sus participantes, sino que sobre todo examina los efectos del sistema en su conjunto. Las aplicaciones del análisis de redes sociales pueden ser encontradas en procesos colectivos de tomas de decisiones (Leavitt, 1951) y en relaciones que se dan en una comunidad (Wellman, 1988), entre otros. Nosotros consideramos que el aprendizaje organizacional se genera dentro de las redes sociales que se crean en la empresa, por lo que es clave emplear la perspectiva de red en su estudio porque ayuda a desarrollar una cultura de aprendizaje organizacional (Liebowitz, 2007).

Dentro del análisis de redes sociales destaca la Teoría de las Redes de Aprendizaje (Van der Krogt, 1995, 1998; Poell, Chivers, Van der Krogt y Wildermeersch, 2000) que considera que en toda organización funciona una red de aprendizaje, que recoge cómo se organiza éste en el contexto del trabajo de una empresa. Las personas aprenden en toda organización (de forma organizada o caótica) y la red de aprendizaje sólo representa el modo en el que se organiza el conocimiento. En este trabajo empleamos el concepto de redes sociales para entender la dinámica del aprendizaje organizacional que se produce dentro de una organización.

El aprendizaje organizacional es uno de los temas más relevantes en las recientes investigaciones sobre dirección estratégica (Beckman y Haunschild, 2002; Bapuji y Crossan, 2004; Elkjaer, 2004; Kane y Alavi, 2007; Visser, 2007). Algunos estudios empíricos concluyen que altos niveles de aprendizaje organizacional contribuyen a mejoras en el resultado de la organización (De Geus, 1988; Stata, 1989). Sin embargo, existe una importante laguna en la investigación relacionada con el modo en el que se genera el aprendizaje intraorganizativo, por lo que se comienzan a realizar diversos 
Redes de aprendizaje intraorganizativo: análisis exploratorio de un caso

Sánchez, Jesús D.; Škerlavaj, Miha; Guadamillas Gómez, Fátima y Dimovski, Vlado

estudios de investigación (Škerlavaj, Dimovski, Mrvar y Pahor, 2008; Pahor, Škerlavaj y Dimovski, 2008).

De Geus (1988) establece que la habilidad de aprender más rápido que los competidores puede ser la única fuente de ventaja competitiva sostenible en el tiempo. En esta línea, Škerlavaj, Indihar, Škrinjar y Dimovski (2007) proporcionan apoyo empírico a la afirmación de que altos niveles de aprendizaje organizacional contribuyen a incrementar el valor añadido por empleado, la rentabilidad de los activos, la satisfacción tanto del cliente como del empleado y la calidad de las relaciones con los principales proveedores. Una vez que se corrobora esta relación, una cuestión que surge es cómo se produce dicho aprendizaje.

El aprendizaje organizacional ocurre a múltiples niveles (individual, grupo, organizacional y entre organizaciones) (Sánchez, 2001; Holmquist, 2004; Ibarra et al., 2005; Boh et al., 2006). De este modo, el concepto de aprendizaje organizacional debería ser estudiado como un fenómeno relacional que implica la interacción entre compañeros (Schandt y Marquardt, 2000; Borgatti y Cross, 2003). Estudios previos sobre redes de conocimiento han demostrado que las relaciones de aprendizaje que se producen en las redes no son recíprocas (Borgatti y Cross, 2003). Normalmente, los empleados que son fuente de aprendizaje no son buscadores de conocimiento sino que tienen otro objetivo como es el mejorar su estatus en la organización al contribuir a la generación de aprendizaje (Lazega et al., 2006).

Por su parte, Chan y Liebowitz (2006) y Liebowitz (2007) reconocen el análisis de redes como una herramienta muy útil para la investigación de los flujos de conocimiento que se producen dentro de una empresa. Las redes pueden ayudar a explicar como una idea o conocimiento puede generarse y expandirse a través de los contactos interpersonales. Por tanto, el considerar a una empresa como a una red tiene importantes consecuencias para la gestión de conocimiento de la organización. Así, el responsable de gestión del conocimiento deberá ser consciente de dicha red y emplearla para ayudar a distribuir el conocimiento a través de la organización.

Como ya hemos señalado, nuestra aportación al tema objeto de estudio es analizar el proceso de aprendizaje intraorganizacional considerando que el aprendizaje se genera en las relaciones sociales entre los empleados de una organización. Por tanto, consideramos necesario analizar cuál es el estado de la cuestión en el ámbito del aprendizaje organizacional. Existen dos perspectivas, principalmente, de análisis del aprendizaje organizacional (Cuadro 1):

- Perspectiva de la adquisición: Es la aproximación más tradicional que ha analizado el aprendizaje organizacional (March y Simon, 1958; Cyert y March, 1963; Huber, 1991) y especialmente la organización del aprendizaje (Senge, 1990; Pedler y Aspinwall, 1998). Señala que el aprendizaje es la adquisición individual de conocimiento y habilidades. La mente se considera como un lugar de almacenamiento y el conocimiento como una sustancia, por lo que el aprendizaje se define como la transferencia y acumulación de dicha sustancia en la mente. Esta perspectiva es la dominante porque refleja el entendimiento de un aprendiza- 
Cuadro 1. Perspectivas del aprendizaje organizacional

\begin{tabular}{llll}
\hline & \multicolumn{1}{c}{ Adquisición } & Participación & \multicolumn{1}{c}{ Equilibrio entre ambas } \\
\hline $\begin{array}{l}\text { Objetivo y } \\
\text { contenido del } \\
\text { aprendizaje }\end{array}$ & $\begin{array}{l}\text { Ser un trabajador } \\
\text { habilidoso y con } \\
\text { conocimiento sobre } \\
\text { la organización }\end{array}$ & $\begin{array}{l}\text { Convertirse en un } \\
\text { participante pleno } \\
\text { de habilidades en } \\
\text { la organización }\end{array}$ & $\begin{array}{l}\text { Tener habilidades y conoci- } \\
\text { miento sobre la organización y } \\
\text { convertirse en un participante } \\
\text { pleno de habilidades en la or- } \\
\text { ganización }\end{array}$ \\
$\begin{array}{l}\text { Método del } \\
\text { aprendizaje }\end{array}$ & $\begin{array}{l}\text { Adquisición de } \\
\text { habilidades y } \\
\text { conocimiento }\end{array}$ & $\begin{array}{l}\text { Participación en } \\
\text { comunidades } \\
\text { de práctica }\end{array}$ & $\begin{array}{l}\text { Adquisición de habilidades y } \\
\text { conocimiento y participación } \\
\text { en comunidades de prácticas }\end{array}$ \\
Organización & Sistema & $\begin{array}{l}\text { Comunidad de } \\
\text { prácticas }\end{array}$ & $\begin{array}{l}\text { El proceso de aprendizaje ne- } \\
\text { cesita ser contextualizado } \\
\text { dentro del ámbito de otros pro- } \\
\text { cesos sociales }\end{array}$ \\
\hline
\end{tabular}

Fuente: Adaptado de Elkjaer (2004) y Lazega (2002).

je que deriva de un "sistema de educación formal".

- Perspectiva de la participación: Deriva de estudios empíricos donde se observa una falta de enseñanza formal (Lave y Wenger, 1991). Dentro de esta perspectiva, el aprendizaje se origina a través de la participación en comunidades de prácticas. Esta perspectiva, extrae el aprendizaje de la mente del individuo y de la educación formal y lo localiza en todo aspecto cotidiano que surge día a día en el funcionamiento de cualquier organización. Así, se crean redes cognitivas que ayudan a los miembros de la organización a encontrar compañeros que poseen algún tipo de conocimiento que ellos necesitan. De este modo, al poseer distintos niveles de experiencia y por tanto de conocimientos, existen trabajadores que son mayor fuente de aprendizaje por parte de sus compañeros. Hollingshead (2000), Lewis et al. (2005) y Wegner et al. (1991) sugieren que el nivel de habilidades y experiencia que los em- pleados poseen determinan su capacidad para ser fuente de aprendizaje.

En la literatura se refleja que las dos perspectivas están incompletas a la hora de suministrar un entendimiento pleno sobre el aprendizaje organizacional. Elkjaer (2004) considera que lo adecuado sería una "tercera vía", ya que considera que el contenido y el proceso de aprendizaje no son visibles como un experimento químico y que el aprendizaje tiene lugar como un proceso social, más que como un sistema o sólo una comunidad de prácticas.

La perspectiva en red del aprendizaje intraorganizacional combina ambas aproximaciones en términos de contenido y métodos del aprendizaje. De este modo, desde este enfoque se reconoce al individuo como la fuente primaria y el destino del aprendizaje (perspectiva de adquisición), al tiempo que se asume que el aprendizaje tiene lugar en su origen en una red social (perspectiva de participación). Por tanto, se requiere considerar una teoría estructural más amplia de ac- 
Redes de aprendizaje intraorganizativo: análisis exploratorio de un caso

Sánchez, Jesús D.; Škerlavaj, Miha; Guadamillas Gómez, Fátima y Dimovski, Vlado

ción colectiva (Lazega, 2002) en el contexto del aprendizaje organizacional. Además, la perspectiva en red es sensible al papel que ostenta la organización en promover el aprendizaje. De este modo, se abarca tanto las acciones individuales como las que se producen en el contexto (Berends, Boersma y Weggeman, 2003).

\section{Análisis de las redes de aprendizaje: estudio de un caso}

Para comprender el funcionamiento de las redes de aprendizaje, desarrollaremos un análisis desde la perspectiva de redes en una compañía dedicada al sector de electrónica industrial y de defensa. Dicho análisis se define como "el mapa y la medida de las relaciones y los flujos entre las personas, grupos, organizaciones, ordenadores $u$ otras entidades de procesamiento de información o conocimiento" (Krebs, 2004) y nos proporciona un gráfico y un análisis matemático de una interacción compleja entre personas. Los nudos en las redes son los empleados mientras que los enlaces representan las relaciones o flujos entre los mismos.

A continuación, pasamos a analizar el perfil de la empresa estudiada y a exponer las proposiciones a las que llegamos tras el análisis exploratorio de la empresa.

\subsection{Análisis descriptivo de Tecnobit SL.}

Una de las razones para justificar la falta de estudios que examinen la estructura de las redes de aprendizaje intraorganizativo es la dificultad de obtener da- tos, ya que requiere que los investigadores tengan un acceso importante a los empleados de una organización.

TECNOBIT S.L. tiene 240 trabajadores en su centro productivo de Valdepeñas (Ciudad Real). La empresa fue fundada en 1976 con el nombre de DOI-Ingenieros Asociados y se localizaba en Madrid donde su actividad principal era el control de procesos industriales. En 1981 la empresa creó su planta productiva en Valdepeñas adquiriendo la denominación de Tecnobit. Actualmente, cuenta con cinco líneas de actividad con la siguiente distribución de beneficios -Memoria de Tecnobit, S.L. (2007)-: Aviónica (49\%), Sistemas de Comando y Control (21\%), Sistemas de Simulación (14\%), Optrónica (8\%) y Sistemas de Tecnología de la Información (8\%).

Con el fin de competir en un mercado global, Tecnobit ha diseñado una estrategia de crecimiento agresiva con el objetivo de generar sinergias, especialmente basándose en el conocimiento que la empresa va acumulando. De este modo, se incrementa su valor a través de la diversificación. Esta se ha logrado a través del desarrollo interno y de la adquisición de conocimiento por medio de acuerdos cooperativos y compras a otras empresas (Guadamillas, Donate y Sánchez de Pablo, 2008). Por tanto, es una empresa intensiva en innovación donde el componente tecnológico y la generación de conocimiento tienen un papel fundamental. Este hecho, junto al conocimiento de la empresa por parte de los autores del trabajo, ha motivado la elección de la misma para su estudio.

La organización de la empresa se basa en seis departamentos que se muestran en la Tabla 1. 
Revista Venezolana de Gerencia, Año 15, No. 49, 2010

Tabla 1. Tasa de respuesta

\begin{tabular}{lccc}
\hline \multicolumn{1}{c}{ Departamento } & $\begin{array}{c}\mathbf{N}^{\circ} \text { total de } \\
\text { empleados }\end{array}$ & Respuestas & $\begin{array}{c}\text { Tasa de } \\
\text { respuesta }\end{array}$ \\
\hline (1) Ingeniería de diseño y desarrollo & 75 & 72 & $96 \%$ \\
(2) Producción y post-venta & 58 & 47 & $81,03 \%$ \\
(3) Fabricación & 49 & 39 & $79,59 \%$ \\
(4) Servicios generales & 11 & 7 & $63,64 \%$ \\
(5) Administración & 9 & 4 & $44,44 \%$ \\
(6) Calidad & 7 & 6 & $85,71 \%$ \\
Total & 209 & 175 & $83,73 \%$ \\
\hline
\end{tabular}

Fuente: Elaboración propia.

Comprobamos como los principales departamentos en cuanto al número de trabajadores son los tres primeros, donde por otra parte nos encontramos con la mayor tasa de respuesta. Los otros tres departamentos representan una parte muy reducida de la muestra y su labor, principalmente, es de apoyo a los tres departamentos claves de la empresa.

Otro aspecto relevante a la hora de analizar una red social es el nivel de formación que presentan sus empleados. Formulamos una pregunta donde los trabajadores debían contestar sobre su nivel de formación según 6 categorías. Podemos destacar que un $70,29 \%$ de los empleados posee algún título universitario lo que viene justificado por la alta cualificación que requiere el desarrollo de su actividad empresarial. Por su parte, un reducido $4,57 \%$ sólo posee estudios primarios.

Si analizamos la formación en los principales departamentos, identificamos que aquel que posee mayor nivel de formación es el primero con un 93,06\% de trabajadores con algún título universitario, seguido del segundo con un $57,45 \%$ y por último el tercero con un $41,03 \%$. De este modo, se comprueba que a medida que pasamos de un departamento a otro el nivel de cualificación se reduce, por lo que consideramos que los departamentos con mayor presencia en nuestra muestra son los que requieren mayor nivel de cualificación.

En referencia a la estructura jerárquica de la empresa, agrupamos a los empleados en cuatro categorías: alta dirección $(0,57 \%)$, mandos intermedios $(17,14 \%)$, jefe de proyectos $(5,75 \%)$ y operarios de producción, administración e I+D $(76,57 \%)$. La empresa se organiza por proyectos. En cada uno de ellos existirá un responsable de cada una de las líneas de actividad y un jefe de programa (responsable de la gestión económica, plazos, etc.), un jefe de proyecto (personal técnico) y cuatro especialistas (calidad, pruebas, fabricación y diseño). Entre los especialistas hay un jefe de equipo que responderá ante el jefe de proyectos. Existe alta flexibilidad del personal, ya que se fomenta la rotación entre los diferentes proyectos de las diversas líneas de actividad, tratando de desarrollar una "ingeniería concurrente", es decir, tratar 
Redes de aprendizaje intraorganizativo: análisis exploratorio de un caso

Sánchez, Jesús D.; Škerlavaj, Miha; Guadamillas Gómez, Fátima y Dimovski, Vlado

de que los empleados tengan nociones de la actividad de la empresa en su conjunto (Cuquerella, entrevista 2007).

Entre los tres principales departamentos, destaca el departamento de ingeniería de diseño y desarrollo con un $19,44 \%$ de mandos intermedios y un $9,72 \%$ de directores de proyecto. Por tanto, junto a la mayor cualificación del departamento de ingeniería de diseño y desarrollo, nos encontramos con un mayor porcentaje de altos niveles jerárquicos.

\subsection{Análisis exploratorio de red: establecimiento de proposiciones}

Comenzaremos realizando un análisis de la red de aprendizaje de Tecnobit agrupando a sus empleados por departamentos para poder plantear las dos primeras proposiciones. Posteriormente nos centraremos en conocer cuáles son las características de los empleados que son mayor fuente de aprendizaje para sus compañeros. A continuación, buscaremos subgrupos sociales cohesionados y estudiaremos que características son las que promueven el aprendizaje dentro de los mismos. Para finalizar, analizaremos la importancia de la posición que ocupan los empleados en la red, destacando la relevancia de las posiciones centrales ya que trabajadores que ocupen ese lugar serán claves para los flujos de aprendizaje que se generen en la empresa.

Red de aprendizaje en Tecnobit

Observamos una red interpersonal directa con seis departamentos ubicados en una misma zona geográfica. En la red se representa de que compañeros se aprende (Figura 1). Los departamentos se representan a través de diversas figu- ras geométricas recogidas en la leyenda de la figura.

La empresa propicia e incentiva la cooperación y el desarrollo de relaciones informales entre trabajadores, ya que es conocedora de sus consecuencias positivas: incrementos del valor añadido por empleado, de mejoras en la calidad en las relaciones con los trabajadores y clientes (Dimovski y Škerlavaj, 2004).

La Figura 1 muestra una fuerte conectividad dentro de cada uno de los seis departamentos. Sin embargo, también se producen, aunque en menor número, relaciones de aprendizaje entre departamentos. Cabe destacar que todos los departamentos poseen algún miembro que aprende de algún empleado del de ingeniería de diseño y desarrollo.

Las relaciones entre los tres primeros departamentos son superiores, por una parte, porque tienen mayor número de trabajadores y por otra, porque la efectividad del trabajo de un departamento depende de los resultados de los otros dos. El departamento 1 contiene a los ingenieros de diseño y desarrollo de productos, en el departamento 2 trabajan los técnicos de producción, programadores, ingenieros de pruebas y técnicos de post-venta, mientras que el departamento 3 agrupa a los técnicos de inventarios, técnicos de apoyo a la producción y operarios de tareas propias de la fabricación que requieren menor cualificación. Por tanto se perciben complementariedades en el conocimiento entre los mismos, por lo que se espera que se produzca aprendizaje entre ellos.

Fuentes de aprendizaje: grado de entrada

Uno de los aspectos más importantes de nuestra investigación es analizar 


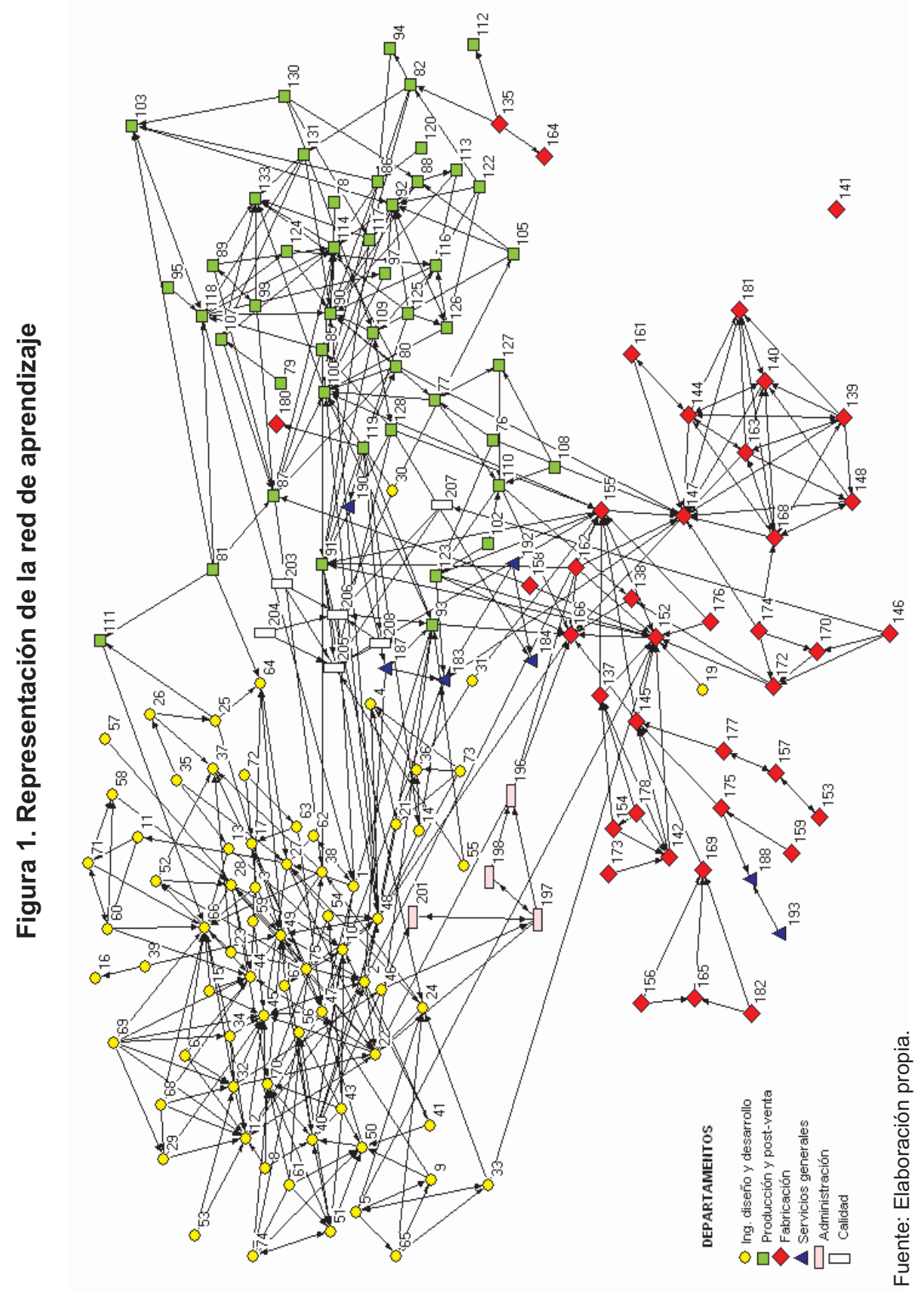


Redes de aprendizaje intraorganizativo: análisis exploratorio de un caso

Sánchez, Jesús D.; Škerlavaj, Miha; Guadamillas Gómez, Fátima y Dimovski, Vlado

cuáles son las características de aquellos empleados que son fuente de aprendizaje de sus compañeros, ya que son éstos los que contribuyen principalmente a la transferencia de conocimiento dentro de la empresa y, por tanto, los que permiten que esta pueda mejorar sus resultados. Nos encontramos en la literatura de redes de aprendizaje con distintas variables que tratan de analizar la importancia de cada empleado en una red. Una de las más empleadas es el grado de entrada (input degree), que representa el porcentaje de compañeros dentro de la organización que aprenden de un trabajador con respecto al total de empleados. En la Tabla 2, recogemos los 10 trabajadores de Tecnobit que se constituyen como principales fuentes de aprendizaje.

Uno de los aspectos a destacar de estos diez trabajadores es que todos poseen un título universitario (el $60 \%$ son licenciados o ingenieros superiores) salvo el empleado 147, aunque el valor medio de la formación académica de estos 10 trabajadores coincide prácticamente con el valor de la muestra.

Con respecto a la posición jerárquica, resaltar que los empleados de los que más se aprende son los mandos intermedios, ya que entre los diez primeros se encuentran un $80 \%$, cuando su importancia con respecto al total es de un $17,14 \%$. Soo-Hoon y Keng-Howe (2000) apuntan que los superiores tratan de que sus subordinados aprendan de ellos para mejorar así su estatus.

Relacionado con el departamento, destacan con un $60 \%$ los trabajadores del departamento de diseño y desarrollo, cuando sólo representan un 41,14\% del total de la muestra. Por su parte, el aspec- to más relevante a destacar es la alta experiencia, tanto en la empresa como en el sector, de estos empleados. Así, nos encontramos con que presentan unas medias de 17,15 años de experiencia en el sector y 161,9 meses en la empresa, las cuales superan ampliamente las medias de toda la muestra.

Por tanto, podemos afirmar que las características comunes de los empleados que son mayor fuente de aprendizaje son: alta posición jerárquica y gran experiencia tanto en la empresa como en el sector. Sólo habría una excepción que es el trabajador con mayores entradas de aprendizaje (ingeniero de software), que presenta una experiencia menor a la media. Por ello podemos establecer las siguientes proposiciones:

P1: A medida que aumenta la experiencia de un trabajador en la empresa, mayor será la probabilidad de que sus compañeros aprendan de él

P2: A medida que aumenta la posición jerárquica de un trabajador mayor será la probabilidad de que sus compañeros aprendan de él.

\section{Análisis de grupos sociales cohe- sivos \\ Hasta ahora hemos analizado la} importancia individual de los empleados en la red de aprendizaje (perspectiva de adquisición). Sin embargo, existen ocasiones en las que se forman subgrupos cohesionados dentro de la red que favorecen un aprendizaje intenso entre los empleados que forman parte de ellos y, a su vez, se transmite al resto de la empresa gracias a las conexiones individuales con el resto de integrantes de la red que tengan sus miembros (perspectiva de 
Tabla 2. Trabajadores con mayor número de entradas de aprendizaje

\begin{tabular}{|c|c|c|c|c|c|c|}
\hline $\begin{array}{l}\text { Código } \\
\text { empleado }\end{array}$ & $\begin{array}{c}\text { Input } \\
\text { degree }\end{array}$ & Departamento & $\begin{array}{c}\text { Experiencia } \\
\text { sector } \\
\text { (años) }\end{array}$ & $\begin{array}{c}\text { Experiencia } \\
\text { empresa } \\
\text { (meses) }\end{array}$ & Educación & Jerarquía \\
\hline 66 & 0.08 & 1 & 7 & 36 & Lcdo./IS & Operario \\
\hline 100 & 0.08 & 2 & 25 & 300 & Dip./IT & Mando intermedio \\
\hline 147 & 0.075 & 3 & 14 & 168 & Bach./FP & Mando intermedio \\
\hline 152 & 0.075 & 3 & 8 & 96 & Dip./IT & Mando intermedio \\
\hline 45 & 0.069 & 1 & 17 & 168 & Lcdo/IS & Jefe de Proyecto \\
\hline 90 & 0.069 & 2 & 5.5 & 66 & Lcdo./IS & Mando intermedio \\
\hline 12 & 0.063 & 1 & 14 & 168 & Lcdo./IS & Mando intermedio \\
\hline 49 & 0.063 & 1 & 15 & 180 & Lcdo./IS & Mando intermedio \\
\hline 22 & 0.057 & 1 & 43 & 168 & Lcdo./IS & Mando intermedio \\
\hline 40 & 0.057 & 1 & 23 & 269 & Dip/IT & Mando intermedio \\
\hline Media 10 & 0.0688 & & 17.15 & 161.9 & & \\
\hline Media 175 & 0.016 & & 7.60 & 65.25 & & \\
\hline \multicolumn{4}{|c|}{ Lcdo./IS = Licenciado/Ingeniero Superior } & \multicolumn{3}{|c|}{ Dip/IT = Diplomado/ Ingeniero Técnico } \\
\hline
\end{tabular}

Fuente: Elaboración Propia.

participación). Existe una alta probabilidad de que entre los trabajadores de los subgrupos existan normas compartidas de comportamiento y se presente una intensa solidaridad entre ellos, porque en definitiva configuran un grupo social dentro de la empresa.

Para detectar estos grupos sociales emplearemos la densidad de la red y subredes. Nuestro objetivo será explorar cuales son las características comunes de los miembros de estos grupos que favorecen el aprendizaje entre compañeros. Consideramos que las semejanzas encontradas dentro de aquellas redes con mayor densidad fomentan el aprendizaje. En este caso, los resultados de este estudio tendrían implicaciones para los gestores, ya que éstos conocerán las variables a evaluar a la hora de organizar a sus empleados para potenciar el aprendizaje organizativo.

En la Tabla 3 se presentan las densidades de la red y las subredes. El valor de la densidad fluctúa entre 0 y 1 . La densidad tomará el valor 1 cuando todos los empleados aprendan de todos y 0 cuando no haya relación entre ellos. De este modo, que la red presente una densidad de 0,016 significa que en la red de aprendizaje de Tecnobit se producen un $1,6 \%$ de todas las relaciones de aprendizaje posibles. Se aprecia, que a medida que aumenta el tamaño de una red se reduce su densidad, lo cual resulta coherente si consideramos, que el número de relaciones que puede mantener una persona es limitado. Aunque no es posible comparar densidades entre redes con distinto tamaño, podemos obtener algu- 
Redes de aprendizaje intraorganizativo: análisis exploratorio de un caso

Sánchez, Jesús D.; Škerlavaj, Miha; Guadamillas Gómez, Fátima y Dimovski, Vlado

nas conclusiones de la evaluación de las densidades.

En la Figura 1, apreciábamos como el aprendizaje que se produce dentro de cada departamento supera ampliamente al producido entre departamentos, por lo que este es un criterio importante en la determinación de grupos sociales cohesionados dentro de la empresa. Normalmente, el hecho de compartir departamento hace que los empleados trabajen en unas mismas instalaciones (oficinas o laboratorios), por lo que es habitual que un trabajador aprenda de aquel que tiene en sus proximidades.

Sin embargo, también encontramos un número importante de relaciones interdepartamentales, que en principio tienen como fin buscar la complementariedad de conocimiento de los empleados. Estas relaciones de complementariedad se hacen más evidentes en las relaciones entre los tres primeros departamentos por las relaciones entre las funciones de sus empleados.

Por su parte, con respecto a la variable experiencia en la empresa hemos clasificado a los trabajadores en dos grupos (experiencia inferior y superior a la media). Encontramos que en el subgrupo de trabajadores con una experiencia superior a la media se incrementan de forma considerable las conexiones de aprendizaje. Así, comprobamos que dicho subgrupo presente una densidad superior a la de la mayor parte de subredes representadas en la Tabla 4 pese a contar con mayor número de trabajadores.

Con respecto a las subredes obtenidas con el criterio de la formación académica nos encontramos que entre los tres grandes grupos (Bachillerato/FP, Di- plomado/lng. Técnico y Licenciado/Ing. Superior) el que presenta mayor número de empleados es a su vez el que obtiene una mayor densidad de red. Sin embargo, las densidades de las subredes no mejoran de forma sustancial los datos obtenidos por la red, como si ocurre con los criterios de división de la experiencia y el departamento.

Uno de los datos más elevados de densidad en relación al tamaño de la subred lo presenta el grupo que conforman los mandos intermedios de la empresa. En este caso, visualizando las gráficas de los diferentes subgrupos obtenidos se percibe que estos empleados son muy importantes en la configuración de la red de aprendizaje de la empresa. Por tanto, las similitudes en cuanto a la posición jerárquica podrían ostentar un papel relevante en la determinación de la red de aprendizaje. Sin embargo, las relaciones entre el grupo de operarios poseen una densidad inferior a la de la red en su conjunto. Si analizamos la variable experiencia dentro de cada grupo, podemos entender que las diferencias en densidad podrían venir determinadas por las mayores semejanzas en el nivel de experiencia en el subgrupo de los mandos intermedios. Por tanto, volvemos a apreciar la importancia de las semejanzas en experiencia para que los empleados aprendan entre ellos.

En función del análisis realizado podemos establecer las siguientes proposiciones:

P3: Las semejanzas en el nivel de experiencia aumentan la probabilidad de que los trabajadores aprendan los unos de los otros

P4: La complementariedad en el conocimiento que se posee aumenta la 
Revista Venezolana de Gerencia, Año 15, No. 49, 2010

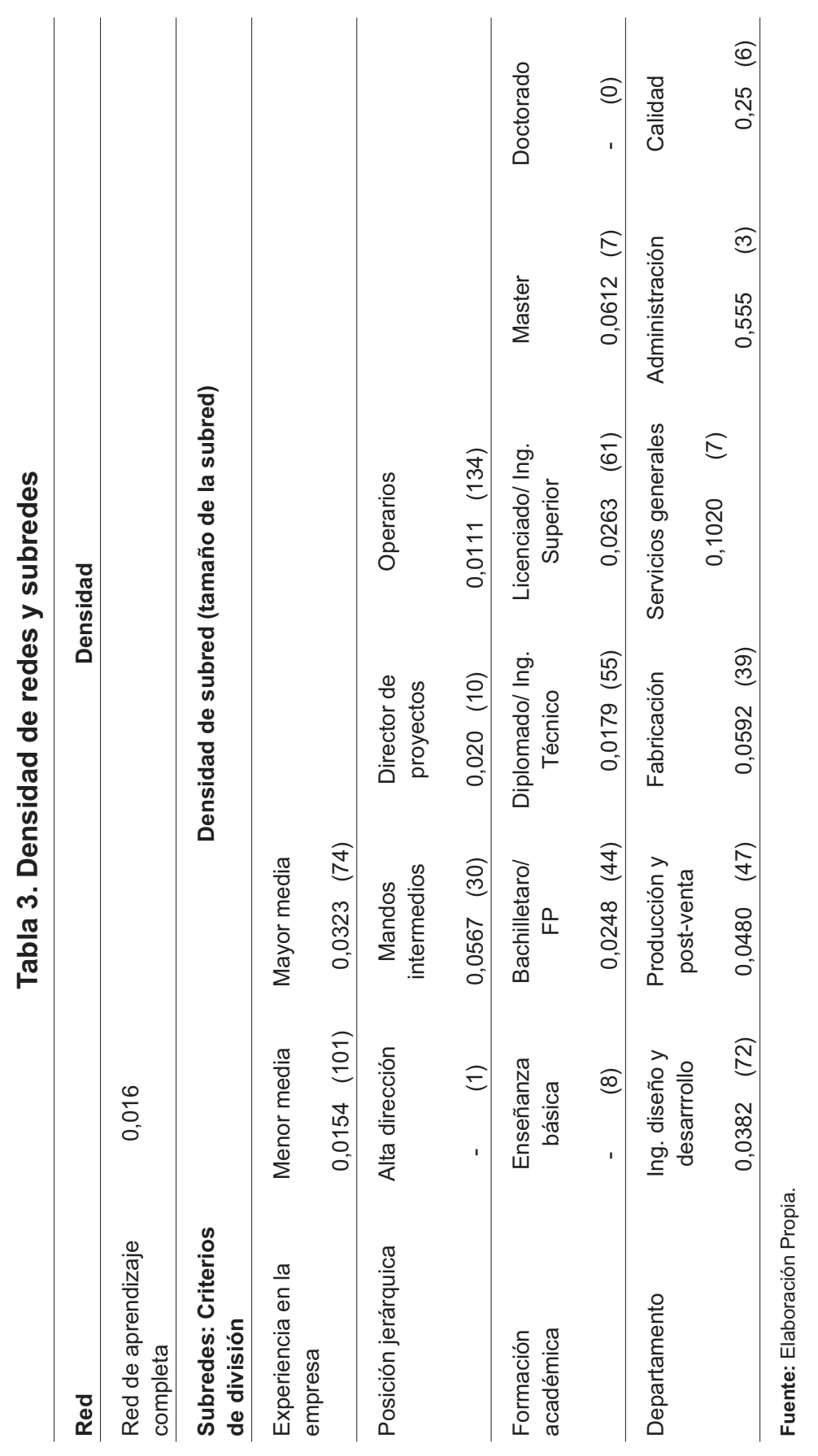


Redes de aprendizaje intraorganizativo: análisis exploratorio de un caso

Sánchez, Jesús D.; Škerlavaj, Miha; Guadamillas Gómez, Fátima y Dimovski, Vlado

probabilidad de que los empleados aprendan los unos de los otros

P5: La proximidad física, determinada por la pertenencia a un mismo departamento, aumenta la probabilidad de que los trabajadores aprendan los unos de los otros diarios.

Medidas de centralidad: interme-

Sin embargo, lo importante de una red de aprendizaje no sólo es el número de contactos que se mantenga en ella, sino la posición de un empleado en la misma. Ésta dependerá del número de relaciones, de la heterogeneidad de los empleados con los que se mantiene relaciones de aprendizaje y de las relaciones que a su vez tienen éstos (Gulati, 1999; Bell, 2005). Uno de los aspectos principales es mantener una posición central en la red, es decir, estar relacionado con un amplio número de trabajadores, lo que favorecerá el aprendizaje.

La perspectiva en red del aprendizaje intraorganizativo señala que aquellos empleados que ostenten un poder central en la red de información podrán asumir labores de intermediarios cuando la empresa quiera negociar algunos aspectos relacionados con su actividad, ya que de este modo se garantiza que la información llegue a un número considerable de empleados, además de conocer las impresiones de estos (Easton, 1992). Estos trabajadores actuarán de intermediarios entre los distintos grupos de la empresa.

Debido a la alta densidad que presentan las distintas subredes según la clasificación del departamento, utilizamos este criterio para analizar los intermediarios. Para identificar a los intermediarios hay que evaluar todas las triadas de vértices que contenga la muestra y analizar dichas relaciones de acuerdo al grupo al que pertenezca cada vértice. En función de esto y siguiendo el enfoque de las redes sociales (Easton, 1992), obtenemos del programa Pajek 1.04 cinco tipos de intermediarios:

- Un coordinador es la persona que conecta dos miembros de un mismo grupo, los cuales no aprenden directamente el uno del otro.

- Un intermediario itinerante es similar al anterior, pero no pertenece al mismo departamento que los otros dos miembros a los que conecta.

- Porteros. Estos empleados aprenden de otro miembro de su departamento y posteriormente transfieren esta información a otro departamento.

- Intermediarios que conectan a otros dos empleados pertenecientes los tres a distintos departamentos. Este es el tipo de intermediario que más potencia el aprendizaje entre grupos distintos.

- Representantes. Obtienen aprendizaje de un empleado de otro departamento para posteriormente transmitírselo a un compañero de departamento.

Analizando las características que más se repiten entre aquellos miembros que asumen en un mayor número de ocasiones la labor de intermediario y, por tanto, ocupan una posición central en la red comprobamos que todos poseen titulación universitaria (salvo un empleado) y que tienen una experiencia en la empresa superior a la media. En la misma línea, Lave y Wenger (1991) señalan que el conocimiento se traslada desde los trabajadores con mayor experiencia hacia los nuevos empleados dentro de las comunidades de prácticas. 
Por tanto, podríamos plantear las siguientes proposiciones:

P6: A medida que aumenta la experiencia de un trabajador en la empresa se incrementa la probabilidad de que ocupe una posición central en la red de aprendizaje.

P7: A medida que se incrementa la formación académica de un empleado aumenta la probabilidad de que ocupe una posición central en la red de aprendizaje de la empresa.

\section{Conclusiones e implicaciones para la dirección}

El análisis de la red de aprendizaje de una empresa española de electrónica industrial y de defensa, desde la perspectiva de redes, ofrece algunas respuestas a la pregunta de cómo se produce el aprendizaje dentro de las organizaciones.

En nuestro caso, los empleados que más a menudo son fuente de aprendizaje para sus compañeros son aquellos que poseen una experiencia en la empresa mayor a la media y que acumulan, por tanto, experiencia y conocimiento (perspectiva de adquisición). Sin embargo, el aprendizaje también se produce en Tecnobit a través de la participación en proyectos e implica la transferencia de conocimiento tácito. Por eso, se recomienda el fomento de los grupos cohesionados, ya que es en estas unidades donde se origina este tipo de aprendizaje (perspectiva de participación).

De este modo, creemos que hemos proporcionado aportaciones de cómo el aprendizaje se puede obtener a través de distintos modos, que aúnan las corrientes teóricas existentes, es decir, es cierto que el aprendizaje se produce por la participación en comunidades de prácticas, pero también lo es que se genera por el acceso a flujos de conocimiento que se poseen dentro de la organización.

A partir de los resultados del caso analizado, hemos establecido varias proposiciones de investigación, que requieren un mayor análisis en casos de empresas de otros países, otras industrias o incluso de otro tamaño. Las principales aportaciones de nuestro estudio son:

- Se proporciona apoyo a la noción de que la experiencia de los empleados contribuye en su nivel de conocimiento y que el resto de trabajadores serán más propensos a aprender de los empleados con mayor experiencia.

- Relación similar se produce con los empleados situados en posiciones jerárquicas altas, ya que entre los principales empleados que son fuente de aprendizaje se sitúan un número considerable de mandos intermedios.

- La proximidad física (pertenecer al mismo departamento), semejanzas en el nivel de experiencia y complementariedad en el conocimiento son las tres variables identificadas como facilitadoras del aprendizaje dentro de los grupos cohesionados.

- El trabajo ofrece herramientas para detectar los trabajadores más importantes desde el punto de vista del aprendizaje.

Por lo anterior, consideramos que los encargados del departamento de recursos humanos en las empresa tratarán de buscar empleados con experiencia en el sector y de un importante nivel jerárquico conocedores de su importancia en el proceso de aprendizaje organizativo, y por tanto, en el resultado empresarial. 
Redes de aprendizaje intraorganizativo: análisis exploratorio de un caso

Sánchez, Jesús D.; Škerlavaj, Miha; Guadamillas Gómez, Fátima y Dimovski, Vlado

Hay que resaltar que la alta cualificación de la plantilla y su carácter de empresa intensiva en conocimiento e innovación son hechos diferenciales respecto a otras empresas. Estas características provocan que se comparta más conocimiento y se intensifiquen los procesos de aprendizaje para resolver problemas e innovar. Por tanto, sería conveniente corroborar si las relaciones encontradas en esta empresa se producen de igual modo en otras con diferentes características.

Otra aportación relevante de este trabajo son las implicaciones gerenciales que de sus resultados se obtienen. Consideramos que los directivos tienen medios suficientes para influir en las relaciones de aprendizaje intraorganizativas a través de diversos elementos. El primer paso será visualizar toda la red de relaciones existentes en la organización con el fin de identificar los empleados clave (tanto por número de relaciones de aprendizaje como por ocupar una posición central en la red), ya que a través de ellos se puede influir sobre la red en su conjunto. Así, los gerentes podrán: a) desarrollar un ajuste entre la estructura organizacional formal e informal; b) desarrollar y combinar mejores sistemas de recompensa y motivacionales; c) ayudar a entender la cultura organizacional de la compañía; d) ajustar su estilo directivo; y e) ayudar a organizar los programas formativos y educacionales para que las características que presentan dichos trabajadores puedan generalizarse al resto de la organización.

Para concluir señalar que es necesario desarrollar futuras investigaciones donde apliquemos las redes de aprendizaje a otras organizaciones para encon- trar apoyo adicional a las proposiciones mostradas. Una aportación importante podría ser aplicar un análisis similar en empresas de diversos tamaños, diferentes industrias o incluso de diversos países para poder controlar el impacto de dichas variables de contexto. Por ejemplo, consideramos que la cultura organizacional y nacional, así como la propia estructura jerárquica de la empresa también influirán en el aprendizaje generado en las redes sociales producidas en el seno de una empresa, por lo que habrá que controlar sus efectos. De este modo, una probable línea de investigación será analizar redes intraorganizativas de empresas latinoamericanas ya que presentan características culturales similares a las españolas, pudiendo desarrollarse un estudio comparativo entre ambas con el objetivo de poder conocer "mejores prácticas" para potenciar el aprendizaje y facilitar la labor empresarial en el entorno económico actual.

\section{Referencias Bibliográficas}

Bapuji, Hari y Crossan, Mary (2004). Reviewing organizational learning research - From questions to answers. Management Learning, Vol. 35, pp. 397-417.

Batagelj, Vladimir y Mrvar, Andrej (2005). Pajek 1.04 - Program for Large Network Analysis. http://vlado.fmf.unil..si/pub/networks/pajek (Acceso: 08. 02. 2008).

Beckman, Christine y Haunschild, Pamela (2002). Network learning: The effects of partners' heterogeneity of experience on corporate acquisitions. Administrative Science Quarterly, Vol. 47, pp. 92-124. 
Bell, Geoffrey (2005). Clusters, Networks, and Firm Innovativeness. Strategic Management Journal, Vol. 26, pp. 287-295.

Berends, Hans; Boersma, Kees y Weggeman, Mathieu (2003). The structuration of Organizational Learning. Human Relations, Vol. 56, No 9, pp.1035-1056.

Boh, Wai Fong; Slaughter, Sandra A. y Espinosa, J. Alberto (2006). Learning from Experience in Software Development: A Multilevel Analysis. Management Science, Vol. 53, pp. 1315-1332.

Borgatti, Stephen P. y Cross, Rob (2003). A Relational View of Information Seeking and Learning in Social Networks. Management Science, Vol. 49, pp. 432-445.

Chan, Kim y Liebowitz, Jay (2006). The synergy of social network analysis and knowledge mapping: a case study. Int. J. Management and Decision Making, Vol. 7, pp. 19-35.

Cyert, Richard y March, James (1963). A Behavioral Theory of the Firm. Prentice-Hall, Englewood Cliffs, NJ.

De Geus, Arie (1988), Planning as learning. Harvard Business Review, Vol. 88, pp. $70-74$.

De Nooy, Wouter.; Mrvar, Andrej y Batagelj, Vladimir (2005). Exploratory social network analysis with Pajek. Cambridge University Press, New York.

Dimovski, Vlado y Škerlavaj, Miha (2004). Organizational Learning and Its' Impact on Financial and Non-financial Performance. Proceedings of $5^{\text {th }}$ Organizational knowledge, Learning and Capabilities conference, Innsbruck.

Easton, Gary (1992). Industrial Network - A Review, en Axelsson, B. y Easton, G (dir.), Industrial Networks - A New View of Reality. Palgrave, London.

Elkjaer, Bente (2004). Organizational Learning - The Third Way". Management Learning, Vol. 35, No 4, pp. 419-434.
Guadamillas, Fátima; Donate, Mario Javier y Sánchez de Pablo, Jesús David (2008). Knowledge Management for Corporate Entrepreneurship and Growth: A Case Study. Knowledge and Process Management, Vol. 15, pp. 32-44.

Gulati, Ranjay (1999). Network Location and Learning: the Influence of Network Resources and Firm Capabilities on Alliance Formation. Strategic Management Journal, Vol. 20, pp. 397-420.

Hollingshead, Andrea B. (2000). Perceptions of expertise and transactive memory in work relationships. Group Processes and Intergroup Relations, Vol. 3, pp. 257-267.

Holmquist, Mats (2004). Experimental Learning Processes of Exploitation and Exploration Within and Between Organizations: An Empirical Study of Product Development. Organization Science, Vol. 15, pp. 15-32.

Huber, George (1991). Organizational learning: The contributing processes and the literature. Organization Science, Vol. 2, pp. 88-115.

Ibarra, Herminia; Kilduff, Martin y Tsai, Wenpin (2005). Zooming In and Out: Connecting Individuals and Collectivities at the Frontiers of Organizational Network Research. Organization Science, Vol. 16, pp. 359-374.

Kane, Gerald C. y Alavi, Maryam (2007). Information Technology and Organizational Learning: An Investigation of Exploration and Exploitation Processes. Organization Science, Vol. 18, pp. 796-815.

Krebs, Valdis (2004). An Introduction to Social Network Analysis. http://www.orgnet.con/sna.html (acceso: 07.04.2005).

Lave, Jean y Wenger, Etienne (1991). Situated Learning: Legitimate Peripheral Participation. Cambridge University Press, Cambridge. 
Redes de aprendizaje intraorganizativo: análisis exploratorio de un caso

Sánchez, Jesús D.; Škerlavaj, Miha; Guadamillas Gómez, Fátima y Dimovski, Vlado

Lazega, Emmanuel (1992). The Micropolitics of Knowledge. A. de Gruyter, New York.

Lazega, Emmanuel; Lemercier, Claire y Mounier, Lise (2006). A spinning top model of formal organization and informal behaviour: dynamics of advice networks among judges in a commercial court, European Management Review, Vol. 3, pp. 113-122.

Leavit, Harold (1951). Some Effects of Certain Communication Patterns on Group Performance. The Journal of Abnormal and Social Psychology, Vol. 46, No1, pp. 38-50.

Lewis, Kile; Lange, Donald y Gillis, Lynette (2005). Transactive Memory Systems, Learning, and Learning Transfer. Organization Science, Vol. 16, pp. 581-600.

Liebowitz, Jay (2007). Developing knowledge and learning strategies in mobile organisations. International Journal Mobile Learning and Organizations, Vol. 1, No. 1, pp. 5-14.

March, James G. y Simon, Herbert A. (1958). Organizations. Wiley, New York.

Mitchell. J. Clyde (1969). The Concept and Use of Social Networks, en Mitchell, J.C. (dir), Social Networks in urban situations. University of Manchester Press, Manchester.

Pahor, Marko; Škerlavaj, Miha y Dimovski, Vlado (2008). Evidence Supporting the Network Perspectiva on Organizacional Learning. Journal of the American Society for Information Science and Technology, Vol. 12, pp. 1985-1994.

Pedler, Mike y Aspinwall, Kath (1998). A Concise Guide to the Learning Organization. Lemos \& Crane, London.

Poell, Rob F.; Chivers, Geoff; Van der Krogt, Ferd J. y Wildermeersch, Danny A. (2000). Learning-network Theory Organizing the Dynamic Rela- tionships between Learning and Work. Management Learning, Vol. 31, No 1, pp. 25-49.

Sanchez, Ron (2001). Knowledge Management and Organizational Competence. Oxford, University Press, UK.

Schwandt, David y Marquardt, Michael (2000). Organizational learning: From Worldclass Theories to Global Best Practices. St Lucie, Boca Raton.

Senge, Peter M. (1990). The Fifth Discipline The Art and Practice of the Learning Organization. Doubleday Currency, Nueva York.

Škerlavaj, Miha; Indihar Štemberger, Mojca; Škrinjar, Rok y Dimovski, Vlado. (2007). Organizational learning culture-the missing link between business process change and organizational performance. International Journal of Production Economics, Vol. 106, pp. 346-367.

Škerlavaj, Miha; Dimovski, Vlado; Mrvar, Andrej y Pahor, Marko (2008). Intra-organizational learning networks within knowledge-intensive learning environments, Interactive Learning Environments, on line, DOI: $10.1080 /$ 10494820802190374

Soo-Hoon, Lee y Phan, Chew Irene (2006). The impact of social networks on pay increase, promotions and layoffs. Labor and Management Development Journal, Vol.1, No. 9, pp. 1-15.

Stata, Raymie (1989). Organizational learning: The key to management innovation. Sloan Management Review, Vol. 30, pp. 63-74.

Tichy, Noel; Tushman, Michael y Fombrun, Charles (1979). Social Network Analysis for Organizations. Academy of Management Review, Vol.4, No 4, pp. 507-519.

Van der Krogt, Ferd J. (1995). Leren in Netwerken: veelzijdig organiseren van leernetwerken met het oog ophumaniteit 
en arbeidsrelevantie. Lemma, Utrecht.

Van der Krogt, Ferd J. (1998). Learning Network Theory - The Tension between Learning Systems and work Systems in Organizations. Human Resource Development Quarterly, Vol.9, No 2, pp. 157-177.

Visser, Max (2007). Deutero-learning in organizations: A review and a reformulation. Academy of Management Review, Vol. 32, pp. 659-670.

Wasserman, Stanley y Faust, Katherine (1994). Social Network Analysis. Cambridge University Press,Cambridge, UK.
Wegner, Daniel M.; Erber, Ralph y Raymond, Paula (1991). Transactive memory in close relationships. Journal of Personality and Social Psychology, Vol. 61, pp. 923-929.

Wellman, Barry (1988). Structural analysis: From method and metaphor to theory and substance. B. Wellman, S. D. Berkowitz, eds. Social Structures: A Network Approach. Cambridge University Press, New York.

\section{Entrevista:}

Cuquerella, Antonio. Director de Producción y Director de Planta Productiva de Valdepeñas; Valdepeñas 2 de noviembre de 2007. 